\title{
ON PREFRATTINI RESIDUALS ${ }^{\dagger}$
}

\section{by A. BALLESTER-BOLINCHES, H. BECHTELL and L. M. EZQUERRO}

(Received 23 September, 1996)

Introduction. All groups considered in the sequel are finite. Let $\mathfrak{C}$ and $\mathfrak{I}$ denote the formations of groups which consist of collections of groups that respectively either split over each normal subgroup (nC-groups) or for which the groups do not possess nontrivial Frattini chief factors [8]. The purpose of this article is to develop and expand a concept that arises naturally with the residuals for these formations, namely each $G$-chief factor $G^{\mathbb{C}} / \mathrm{K}(\mathrm{G} \mathfrak{J} / \mathrm{K})$ is non-complemented (Frattini). With respect to a solid set $\mathbf{X}$ of maximal subgroups, these properties are generalized respectively to so-called $\mathbf{X}$-parafrattini (X-profrattini) normal subgroups for which each type is closed relative to products. The relationships among the unique maximal normal subgroups that result from these products, the solid set of maximal subgroups $\mathbf{X}, \mathbf{X}$-prefrattini subgroups, and the residuals of formations are explored. This leads to a well-defined collected of formations, the partially nonsaturated formations, with properties analogous to those which are totally non-saturated. In the development, attention is given to a set of maximal subgroups which is the image of a solid function defined on all groups, a weaker condition than that of a solid set. A result of particular interest answers affirmatively the long-standing conjecture that a non-trivial nC-group $G$ is solvable if and only if each $G$-chief factor is complemented by a maximal subgroup. This will force a critical re-examination of the classification problem for $\mathrm{nC}$-groups. Since the article continues the investigations on finite groups initiated in [2], a familiarity with that article is assumed. All other notation and terminology is from [6]. If $M$ is a maximal subgroup of a group $G$ and $G / C$ or $e_{G}(M)$ is a monolithic primitive group, i.e. a group with a unique minimal normal subgroup, then $M$ is called a monolithic maximal subgroup of $G$.

1. X-pro(-para) frattini subgroups. Definitions. Given a set $\mathbf{X}$ of maximal subgroups of a group $G$, we say that a chief factor $H / K$ of $G$ is

(i) an $\mathbf{X}$-Frattini chief factor of $G$ if no maximal subgroup in $\mathbf{X}$ supplements $H / K$;

(ii) a non-X-complemented chief factor of $G$ if no maximal subgroup in $\mathrm{X}$ complements $H / K$;

(iii) an $\mathbf{X}$-supplemented chief factor of $G$ if $\mathrm{H} / \mathrm{K}$ has a supplement in $\mathbf{X}$;

(iv) an $\mathbf{X}$-complemented chief factor of $G$ if $\mathrm{H} / \mathrm{K}$ has a complement in $\mathbf{X}$.

Definitions. [2] (i) A set $\mathbf{X}$ of monolithic maximal subgroups of a group $\mathrm{G}$ is said to be $J H$-solid if it satisfies the following condition:

(JH) If $M_{1}, M_{2} \in \mathrm{X}$ with $C_{1}=\operatorname{Core}_{G}\left(M_{1}\right) \neq \operatorname{Core}_{G}\left(M_{2}\right)=C_{2}$ and both complement an abelian chief factor $H / K$ of $G$ then there exists $M \in \mathbf{X}$ such that $\operatorname{Core}_{G}(M)=$ $\left(C_{1} \cap C_{2}\right) H$.

\footnotetext{
†The first author is supported by Proyecto PB 94-0965 of DGICYT, Ministerio de Educación y Ciencia of Spain.
} The third author is supported by Proyecto PB 94-1048 of DGICYT, Ministerio de Educación y Ciencia of Spain.

Glasgow Math. J. 40 (1998) 187-197. 


\section{A. BALLESTER-BOLINCHES, H. BECHTELL AND L. M. EZQUERRO}

(ii) A set $\mathbf{X}$ of maximal subgroups of the group $G$ is said to be solid if it satisfies.

(*) If $M_{1}, M_{2} \in \mathrm{X}$ with $C_{1}=$ Core $_{G}\left(M_{1}\right) \neq$ Core $_{G}\left(M_{2}\right)=C_{2}$ and both complement an abelian chief factor $H / K$ of $G$ then $M=\left(M_{1} \cup M_{2}\right) H \in \mathrm{X}$, and whenever a chief factor is $\mathrm{X}$ supplemented then all its supplements are in $\mathbf{X}$.

REMARK. Let $\mathbf{X}$ be a solid set of maximal subgroups of the group $G$. From [2, paragraph 1, p. 267] one concludes that the set $\mathbf{X}_{\text {mon }}$ of all monolithic maximal subgroups of $G$ in $\mathbf{X}$ is $\mathrm{JH}$-solid. Moreover, if $H / K$ is an $\mathbf{X}$-supplemented chief factor of $G$, then $H / K$ is $\mathbf{X}_{\text {mon }^{-}}$ supplemented. For suppose that $H / K$ is $\mathbf{X}$-supplemented non-abelian chief factor of $G$. Denote $C=C_{G}(H / K)$. Then $G / C \in \mathcal{P}_{2}$ and $\operatorname{Soc}(G / C)=H C / C$. Let $M$ be a maximal subgroup of $G$ supplementing $H C / C$. Then $M$ is a monolithic supplement of $H / K$ in $G$. Since $\mathbf{X}$ is solid, the subgroup $M$ belong to $\mathbf{X}$. In other words $M \in \mathbf{X}_{\text {mon }}$.

In view of this observation, a discussion on solid sets can be restricted to $\mathbf{X}_{\text {mon }}$. Consequently in this sense, Theorem A [2] on JH-solid sets is valid for a solid set $\mathbf{X}$.

Definition. Given a solid set $\mathbf{X}$ of maximal subgroups of a group $G$, the subgroup $\Phi_{\mathrm{X}}(G)=\cap\{M: M \in \mathbf{X}\}$ is called the $X$-Frattini subgroup of $G$.

From the Remark, $\Phi_{\mathrm{X}}(G)=\cap\left\{M: M \in \mathbf{X}_{\text {mon }}\right\}$.

From the definition it follows that if $\mathbf{X}$ is a solid set of maximal subgroups of a group $G, N$ is a normal subgroup of $G$ and $\mathbf{X} / N=\{M / N \leq G / N: M \in \mathbf{X}, N \leq M\}$, then $\mathbf{X} / N$ is a solid set of maximal subgroups of $G / N$ and $\Phi_{\mathrm{X}}(G) N / N \leq \Phi_{\mathrm{X} / N}(G / N)$.

Definition. Let $G$ be a group and suppose that $\mathbf{X}$ is a solid set of maximal subgroups of $G$. A normal subgroup $N$ of $G$ is said to be

(i) an X-profrattini normal subgroup of $G$ if either $N=1$ or every chief factor of $G$ of the form $N / K$ is an X-Frattini chief factor of $G$; in other words $N / K \leq \Phi_{\mathrm{X}}(G / K)$ and

(ii) an $X$-parafrattini normal subgroup of $G$ if either $N=1$ or every chief factor of $G$ of the form $N / K$ is a non-X-complemented chief factor of $G$, that is, no maximal subgroup in $\mathbf{X}$ is a complement of $N / K$ in $G$.

If $\mathbf{X}=\operatorname{Max}(G)$, the solid set of all maximal subgroups of $G$, we write simply profrattini and parafrattini.

EXAmples and Remarks. 1. If $N$ is an X-profrattini normal subgroup of $G$, then $N$ is an $\mathbf{X}$-parafrattini normal subgroup of $G$. The converse does not hold in general. it is enough to consider a non-abelian simple group $S$. It is clear that $S$ is $\mathbf{X}$-parafrattini for all solid sets $\mathbf{X}$ of maximal subgroups of $S$. However $S$ is not X-profrattini.

If $N$ is soluble, $N$ is $\mathbf{X}$-profrattini if and only if $N$ is $\mathbf{X}$-parafrattini.

2. If $\mathfrak{F}$ is a totally nonsaturated formation (see [1]), then $G^{\mathfrak{F}}$ is a profrattini normal subgroup of $G$ for every group $G$.

3. A quasinilpotent normal subgroup $N$ of a group $G$ is $\mathbf{X}$-profrattini if and only if $N \leq \Phi_{\mathrm{X}}(G)$.

Proof. Assume that $N$ is a quasinilpotent $\mathrm{X}$-profrattini normal subgroup of $G$ but $N \not \Phi_{\mathrm{X}}(G)$. Then $K=N \cap \Phi_{\mathrm{X}}(G) \neq N$. Since $\Phi_{\mathrm{X}}(G) / K=\Phi_{\mathrm{X}}(G / K)$ and $N / K \nsubseteq \Phi_{\mathrm{X}}(G / K)$, there exists a maximal subgroup $U$ of $G$ such that $K \leq U, U \in \mathbf{X}$ and $G=U N$. Denote $U_{G}=\operatorname{core}_{G}(U)$ and consider the primitive group $G / U_{G}$. Then $G / U_{G}=\left(N U_{G} / U_{G}\right)\left(U / U_{G}\right)$. 
Since $N U_{G} / U_{G}$ is quasinilpotent, it follows that $N U_{G} / U_{G} \leq F^{*}\left(G / U_{G}\right)=\operatorname{Soc}\left(G / U_{G}\right)$, where $F^{*}\left(G / U_{G}\right)$ denotes the generalized Fitting subgroup of $G / U_{G}$. Suppose that $N U_{G} / U_{G}=\operatorname{Soc}\left(G / U_{G}\right)$ and $G / U_{G}$ is a primitive group with two minimal normal subgroups that we denote by $A_{i} / U_{G}, i=1,2$. Since $N$ is $\mathrm{X}$-profrattini, $U$ has to supplement both $A_{1} / U_{G}$ and $A_{2} / U_{G}$. But since $\mathbf{X}$ is solid, there exists a monolithic maximal subgroup $V \in \mathbf{X}$ such that $V$ supplements exactly one of them, say $A_{1} / U_{G}$. This implies $A_{2} \leq V_{G}$. So $K \leq V_{G}$. In other words, we can always choose a maximal subgroup $U$ in $\mathbf{X}$ in such a way that $N U_{G} / U_{G}$ is a chief factor of $G$. But this contradicts $N$ being X-profrattini. Hence $N \leq \Phi_{\mathrm{X}}(G)$. The converse holds trivially.

THEOREM 1.1. Let $G$ be a group and suppose that $X$ is a solid set of maximal subgroups of $G$.

(i) If $N, M$ are both $\mathbf{X}$-profrattini normal subgroups of $G$, then $N M$ is an $\mathbf{X}$-profrattini normal subgroup of $G$.

(ii) If $N, M$ are both $\mathbf{X}$-parafrattini normal subgroups of $G$, then $N M$ is an $\mathbf{X}$-parafrattini normal subgroup of $G$.

Proof. (i) Suppose that a $G$-chief factor $(N M) / K$ is $\mathbf{X}$-supplemented by $S$. Then $G=S(N M)$ and $K \leq S \cap N M$. It is clear that $M, N \neq 1$. If $N \leq K$, then $M N=K M$ and $M /(M \cap K)$ is a chief factor of $G$. Moreover $G=S(N M)=S M$ and $M \cap K \leq K \leq S$. This implies that $S$ is an $\mathrm{X}$-supplement of $M /(M \cap K)$ in $G$, a contradiction. Now $N K / K$ is a normal subgroup of $G / K$ contained in $M N / K$. Hence $M N=N K$. Apply Lemma 1 of [2] with $U=K, V=N, Z=U V=M N=N K$ and $W=U \cap V=K \cap N$. Since $Z / U$ is supplemented in $G$ by $S$, then $V / W=N(K \cap N)$ is supplemented in $G$ by $S$. This contradicts $N$ being X-profrattini.

(ii) Suppose that a $G$-chief factor $(N M) / K$ is $\mathrm{X}$-complemented by $S$. Then $G=S(N M)$ and $K=S \cap N M$. Again we have $N \neq 1$. If $N \leq K, M N=K M$. So $M /(M \cap K)$ is a chief factor of $G$. Now $G=S(N M)=S M$ and $M \cap K \leq K \leq S$. So $M \cap K \leq S \cap M$. On the other hand, $K=S \cap N M=N(S \cap M)=N(K \cap M)$. Hence $|S \cap M|=|K \cap M|$ and $S \cap M=$ $K \cap M$. In particular, $S$ is an X-complement of $M /(M \cap K)$ in $G$. This contradicts the fact that $M$ is an X-parafrattini normal subgroup of $G$. Therefore $M N=N K$. Consider $U=K, V=N, Z=U V=M N=N K$, and $W=U \cap V=K \cap N$ in Lemma 1 of [2]. Since $Z / U$ is complemented in $G$ by $S \in \mathbf{X}$, so is $V / W=N /(K \cap N)$. This contradicts $N$ being $\mathbf{X}$ parafrattini.

Remark. Let $G$ be a group and $\mathbf{X}$ be a solid set of maximal subgroups of $G$. Suppose that $N$ is a normal subgroup of $G$ satisfying the property that either $N=1$ or every chief factor $N / K$ of $G$ is $\mathrm{X}$-complemented in $G$. If $M$ is a normal subgroup of $G$ with the same property, then $M N$ does not have this property in general. For instance, consider $G=A \times B$ where $A=\left\langle a: a^{4}=1\right\rangle, B=\left\langle b: b^{2}=1\right\rangle$, and $\mathbf{X}=\operatorname{Max}(G)$. Then $C=\langle b\rangle$ and $D=\left\langle a^{2} b\right\rangle$ are two complemented minimal normal subgroups of $G$. However $C D=\left\langle a^{2}\right\rangle\langle b\rangle$ has the Frattini chief factor $C D / C$.

Definitions. Let $G$ be a group and $\mathbf{X}$ be a solid set of maximal subgroups of $G$.

(i) The $X$-profrattini subgroup of $G$ is the normal subgroup

$$
\operatorname{Pro}_{\mathbf{x}}(G)=\langle N: N \text { is an } \mathbf{X} \text {-profrattini normal subgroup of } G\rangle \text {. }
$$


190 A. BALLESTER-BOLINCHES, H. BECHTELL AND L. M. EZQUERRO

(ii) The $X$-parafrattini subgroup of $G$ is the normal subgroup

$\operatorname{Para}_{\mathrm{x}}(G)=\langle N: N$ is an X-parafrattini normal subgroup of $G\rangle$.

If $\mathbf{X}=\operatorname{Max}(G)$, the solid set of all maximal subgroups of $G$, we write simply $\operatorname{Pro}(G)$ and $\operatorname{Para}(G)$.

It is clear that $\operatorname{Prox}_{X}(G) \leq \operatorname{Para}_{X}(G)$ and if $X$ is a solid set of maximal sub-groups of $G$ composed of maximal subgroups of type 1 , then $\operatorname{Prox}_{x}(G)=\operatorname{Parax}_{X}(G)$. In particular, the equality holds when $G$ is soluble. However, there exists non-soluble groups $G$ for which $\operatorname{Prox}_{X}(G)=\operatorname{Parax}(G)$. Consider a prime $p$ and a cyclic group $Z$ of order $p^{2}$. Let $G=S: Z$ be the regular wreath product of $S$ with $Z$, where $S$ is a non-abelian simple group. Then $\operatorname{Pro}(G)=\operatorname{Para}(G)$ is the unique maximal normal subgroup of $G$.

It is clear that for each normal subgroup $\left.N>\operatorname{Parax}_{(G)(\operatorname{resp} .} N>\operatorname{Prox}_{(}(G)\right)$ there is at least one $G$-chief factor $N / K$ which is $\mathbf{X}$-supplemented (resp. X-complemented) in $G$. We can say much more than this.

Proposition 1.2. Let $G \in \mathcal{P}_{2}$ split over $\operatorname{Soc}(G)=N$ by a maximal subgroup $S$ of $G$. Then $\operatorname{Soc}(S)$ is nonabelian.

Proof. Let $A$ be an abelian minimal normal subgroup of $S$. Then $A$ is an elementary abelian $p$-group for some prime $p$. Since $S \leq N_{G}(A)$, then $N_{G}(A)=S$ since proper containment leads to a contradiction that $A$ is normal in $G$, by maximality of $S$ in $G$. Hence $N \cap C_{G}(A)=1$. If $p$ divides $|N|$, a contradiction arises since $A$ would be contained in a Sylow $p$-subgroup $P=[T] A$ of $N A$ with $T=P \cap N$. Hence $T$ would contain an element $x \in Z(P) \cap C_{N}(A)=1$. Consequently $p$ does no divide $|N|$. Let $q$ be a prime dividing $|N|$. By Theorem 6.2.2 of [7], there exists a unique $A$-invariant Sylow $q$-subgroup $Q$ of $N$. For any element $s \in S, Q^{s}$ is also $A$-invariant. Consequently, $Q=Q^{s}$ and $S \leq N_{G}(Q)$. Since $N \cap S=1, Q$ is not contained in $S$ and so $G=Q S=N S$. This implies $N=Q$, a contradiction.

COROllary 1.3. Denote by $\mathrm{K}$ the class of all groups $G$ such that every chief factor of $G$ is complemented in $G$ by a maximal subgroup of $G$. Then $K \subseteq S$, where $S$ is the class of all soluble groups.

Proof. Suppose that $\mathrm{K}$ is not contained in $\mathrm{S}$ and consider a group of minimal order $G \in K \backslash S$. Then $G \in b(S)$ and $G$ is a primitive group of type 2 . By hypothesis, $N=\operatorname{Soc}(G)$ is a non-abelian minimal normal subgroup which is complemented in $G$ by a core-free maximal soluble subgroup $S$ of $G$. But $\operatorname{Soc}(S)$ abelian contradicts Proposition 1.2.

Proposition 1.4. Let $G$ be a group and $\mathbf{X}$ be a solid set of maximal subgroups of $G$.

(i) Denote by $\mathcal{N}$ the set of all normal subgroups $N$ of $G$ satisfying the property that every chief factor of $G$ between $N$ and $G$ is X-supplemented in $G$. If $N, M \in \mathcal{N}$, then $N \cap M \in \mathcal{N}$.

(ii) Denote by $\mathcal{K}$ the set of all normal subgroups $N$ of $G$ satisfying the property that every chief factor of $G$ between $N$ and $G$ is $\mathbf{X}$-complemented in $G$. If $N, M \in \mathcal{K}$, then $N \cap M \in \mathcal{K}$.

Proof. Consider a chief series of $G$ from $M$ to $M \cap N$.

$$
N \cap M \unlhd \ldots \unlhd M \text {. }
$$


(i) Consider a chief factor $H / K$ of $G$ in $(\beta)$. Then $H N / K N$ is a chief factor of $G$ between $N$ and $G$. Since $N \in \mathcal{N}$ it follows that $H N / K N$ is $\mathbf{X}$-supplemented in $G$ by $S \in \mathbf{X}$, say. This means that $G=S(H N)$ and $K N \leq S \cap N H$. Hence $G=S H$ and $K \leq S \cap H$. So $H / K$ is $\mathbf{X}$ supplemented in $G$ by $S$. Therefore (i) follows from Theorem A of [2].

(ii) Notice that by Corollary 1.3 , the groups $G / N$ and $G / M$ are soluble. Then $G /(N \cap M)$ is soluble. Therefore all chief factors in $\beta$ are abelian.

Consider a chief factor $H / K$ of $G$ in $(\beta)$. Then $H N / K N$ is a chief factor of $G$ between $N$ and $G$. Since $N \in \mathcal{K}$, it follows that $H N / K N$ is $\mathbf{X}$-complemented in $G$ by $S \in \mathbf{X}$, say. This means that $G=S(H N)$ and $K N=S \cap N H$. Hence $G=S H$ and $K=S \cap H$. So $H / K$ is Xcomplemented in $G$ by $S$. Therefore (ii) follows from Theorem A of [2].

COROLlaRY 1.5. Let $G$ be a group and $\mathbf{X}$ a solid set of maximal subgroups of $G$.

Then

(i) $\operatorname{Prox}(G)=\bigcap\{N: N \in \mathcal{N}\} \in \mathcal{N}$ and every chief factor of $G$ between $\operatorname{Pro}_{x}(G)$ and $G$ is $\mathrm{X}$-supplemented in $G$

(ii) $\operatorname{Para}_{\mathrm{x}}(G)=\bigcap\{N: N \in \mathcal{K}\} \in \mathcal{K}$ and every chief factor of $G$ between $\operatorname{Para}_{\mathrm{x}}(G)$ and $G$ is $\mathrm{X}$-complemented in $G$.

Proof. (i) Denote $K=\bigcap\{N: N \in \mathcal{N}\}$. By Proposition $1.4 K \in \mathcal{N}$. If $K / L$ is an $\mathrm{X}$-supplemented chief factor of $G$, then $L \in \mathcal{N}$ by Theorem A of [2] implies a contradiction. Therefore every chief factor of $G$ of the form $K / L$ is X-Frattini. Hence $K \leq \operatorname{Prox}(G)$. Assume

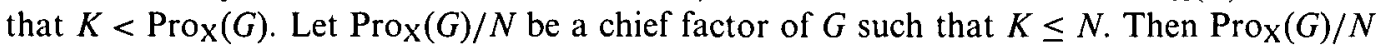
should be X-Frattini. This contradicts Proposition 1.4.

The proof for (ii) is analogous.

COROLlaRY 1.6. If $\mathbf{X}$ is a solid set of maximal subgroups of a group $G$, then $G / \operatorname{Para}_{\mathbf{X}}(G)$ is a soluble group.

Proof. Notice that $G / \operatorname{Parax}_{x}(G) \in K$.

It is clear from the above result that $G^{S}$, the soluble residual of $G$, is contained in $\operatorname{Parax}_{x}(G)$.

COROllary 1.7. If $G$ is a group and $\mathbf{X}$ is a solid set of maximal subgroups of $G$, then $\operatorname{Parax}_{X}(G)=\operatorname{Prox}_{(G)} G^{S}$.

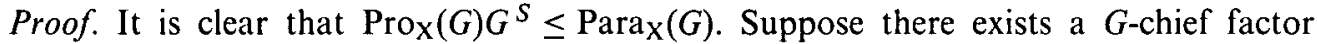

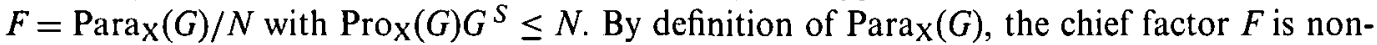
$\mathbf{X}$-complemented in $G$. On the other hand, $F$ is abelian and $\mathbf{X}$-supplemented in $G$ because $\operatorname{Prox}_{(G)} G^{S} \leq N$. Such $F$ cannot exist. So $\operatorname{Parax}(G)=\operatorname{Prox}_{(G)} G^{S}$.

THEOREM 1.8. Let $G$ be a group and $\mathbf{X}$ be a solid set of maximal subgroups of $G$. Then $N$ is an X-parafrattini normal subgroup of $G$ if and only if $N=\left\langle N \cap W^{g}: g \in G\right\rangle$ for each $W \in \operatorname{Pref}(\mathbf{X}, G)$.

Proof. Suppose that $N=\left\langle N \cap W^{g}: g \in G\right\rangle$ for each $W \in \operatorname{Pref}(\mathbf{X}, G)$. Let $N / K$ be a chief factor of $G$. Assume that $N / K$ is $\mathbf{X}$-complemented in $G$. Then there exists a maximal 


\section{A. BALLESTER-BOLINCHES, H. BECHTELL AND L. M. EZQUERRO}

subgroup $M \in \mathbf{X}$ of $G$ such that $G=M N$ and $N \cap M=K$. If $W$ is an $\mathbf{X}$-prefrattini subgroup of $G$ such that $W \leq M, W \cap N \leq M \cap N=K$. Hence $N=\left\langle N \cap W^{g}: g \in G\right\rangle \leq K$, and this is a contradiction. Therefore $\mathrm{N} / \mathrm{K}$ is non-X-complemented in $G$. Hence $N$ is $\mathbf{X}$-parafrattini.

Conversely, assume that $N$ is an $\mathrm{X}$-parafrattini normal subgroup of $G$. We may suppose that $N \neq 1$. Let $W \in \operatorname{Pref}(\mathbf{X}, G)$ and $L=\left\langle N \cap W^{g}: g \in G\right\rangle$. Suppose $L<N$. Let $N / H$ be a chief factor of $G$ such that $L \leq H$. Since $N$ is $\mathbf{X}$-parafrattini we have that $N / H$ is non-Xcomplemented. Notice that $W \cap N \leq L \leq H$ and so $W$ avoids $N / H$. Therefore $N / H$ is $\mathrm{X}$ supplemented. Let $\mathcal{S}$ be the system of maximal subgroups of $G$ such that $W=W(G, \mathbf{X}, \mathcal{S})$ and $M$ be a maximal $\mathbf{X}$-supplement of $N / H$ in $G$ such that $M \in \mathbf{X} \cap \mathcal{S}$. Consider a chief series of $G$ passing through $H$ and $N$. Let $S_{1}, \ldots, S_{r}$ be the $\mathrm{X}$-supplements of the chief factors of $G$ above $N$ such that $S_{i} \in \mathcal{S},(1 \leq i \leq r)$. Then $W N / N=\bigcap_{i=1}^{r} S_{i} / N$ and $W H / H=\bigcap_{i=1}^{r}\left(S_{i} / H\right) \cap$ $(M / H)$. Therefore $W H=\bigcap_{i=1}^{r}\left(S_{i} \cap M\right)=W N \cap M=W(M \cap N)$. Since $W \cap N \cap \stackrel{i=1}{=} W \cap N$ $=W \cap H,|H|=|M \cap N|$. So $H=M \cap N$ and $M$ is an $\mathrm{X}$-complement of $N / H$ in $G$. This contradicts our assumption. Consequently $N$ is an X-parafrattini normal subgroup of $G$.

Given a group $G$ and a solid set $\mathbf{X}$ of maximal subgroups of $G$, the core of each $\mathbf{X}$-prefrattini subgroup of $G$ is $\Phi_{\mathrm{X}}(G)$ (see [2]). The above theorem allows use to describe its normal closure.

Corollary 1.9. Let $G$ be a group and $\mathbf{X}$ be a solid set of maximal subgroups of $G$. If $W \in \operatorname{Pref}(\mathrm{X}, G)$, we have that $W^{G}=\left\langle W^{g}: g \in G\right\rangle=\operatorname{Para}_{\mathrm{x}}(G)$.

Proof. Denote $P=\operatorname{Parax}(G)$. Each abelian chief factor of $G$ which is $\mathbf{X}$-complemented in $G$ is avoided by every $\mathrm{X}$-prefrattini subgroup of $G$. Since every chief factor $H / K$ such that $P \leq K<H \leq G$ is abelian and $\mathrm{X}$-complemented in $G$, it follows that $W \leq \operatorname{Parax}(G)$ for all $W \in \operatorname{Pref}(\mathbf{X}, G)$. From Theorem 1.8, $W^{G}=P$.

\section{Solid functions.}

Definition. A solid function $\chi$ associates to each group $G$ a (possibly empty) solid set $\chi(G)$ of maximal subgroups of $G$ such that

(a) if $\theta: G \rightarrow H$ is an isomorphism, then $\chi\left(G^{\theta}\right)=\left\{M^{\theta}: M \in \chi(G)\right\}$;

(b) if $M \in \chi(G), N \unlhd G$, then $\chi(G / N)=\{M / N: M \in \chi(G), N \leq M\}$.

Equivalently we can say that a solid function $\chi$ associates to each group $G$ a (possibly empty) solid set $\chi(G)$ of maximal subgroups of $G$ such that $\chi\left(G^{\theta}\right)=\left\{M^{\theta}: M \in \chi(G)\right.$, $\operatorname{Ker}(\theta) \leq M$ ) for every epimorphism $\theta \in \operatorname{Epi}(G)$.

The trivial function $\chi_{0}$ defined by $\chi_{0}(G)=\emptyset$ for all groups $G$ is a solid function. The function Max that associates to each group $G$ the set $\operatorname{Max}(G)$ of all maximal subgroups of $G$ is also a solid function. As a result, the collection $\mathcal{C}$ of all solid functions is a lattice with respect to the set-theoretical union and intersection, that is $(\chi \vee \gamma)(G)=\chi(G) \cup \gamma(G)$ and $(\chi \wedge \gamma)(G)=\chi(G) \cap \gamma(G)$ for all groups $G$ and $\chi, \gamma \in \mathcal{C}$.

The next results follows immediately from the definition of solid set. 
COROLlaRY 2.1. (1) The collection $\mathcal{C}$ is a partially ordered modular lattice. The maximal element of $\mathcal{C}$ is the solid function Max; The minimal element is $\chi_{0}$.

(2) The lattice $\mathcal{C}$ is complemented: if $\chi$ is a solid function, then $\chi^{c}$, defined by $\chi^{c}(G)=\operatorname{Max}(G) \backslash \chi(G)$ for each group $G$, is a solid function, and $\chi \bigvee \chi^{c}=\operatorname{Max}$ and $\chi \wedge \chi^{c}=\chi_{0}$.

If $\chi$ is a solid function and $G$ is a group, we denote the $\chi(G)$-Frattini subgroup of $G$ simply by $\Phi_{\chi}(G)$. The concepts of either $\chi$-supplemented or $\chi$-Frattini chief factor are analogous to the previous section. Following the same idea, denote $\operatorname{Pro}_{\chi}(G)=\operatorname{Pro}_{\chi(G)}(G)$ and $\operatorname{Para}_{\chi}(G)=\operatorname{Para}_{\chi(G)}(G)$ for every group $G$.

Let $\chi$ be a solid function. Consider the classes

$F_{\chi}=(G$ : every chief factor of $G$ is $\chi$-supplemented in $G$ or $|G|=1)$

and

$K_{\chi}=(G$ : every chief factor of $G$ is $\chi$-complemented in $G$ or $|G|=1)$.

By Theorem $\mathrm{A}$ of [2], $F_{\chi}$ and $K_{\chi}$ are formations.

If $\chi$ is the solid function defined by $\chi(G)=\operatorname{Max}(G)$ for all groups $G$, then we denote the above classes simply by $\mathrm{F}$ and $\mathrm{K}$ since $G$ belonging to $\mathrm{F}$ is equivalent to not possessing Frattini chief factors.

TheOREM 2.2. (1) $K_{\chi}$ is a formation of soluble groups. Moreover, for each group $G$, we have $\operatorname{Para}_{\chi}(G)=G K_{\chi}$ the $K_{\chi}$-residual of $G$.

(2) Let $K_{\chi}=\left(G: \Phi_{\chi}(G)=1\right)$. Then

$$
F_{\chi}=\left(G: Q(G) \subseteq K_{\chi}\right)=\left(G: \text { for all } N \unlhd G, \phi_{\chi}(G / N)=1\right) .
$$

Moreover, for each group $G$, we have $G^{F_{\chi}}=\operatorname{Pro}_{\chi}(G)$.

(3) $K_{\chi}=F_{\chi} \cap S$.

(4) For every group $G$, we have $G^{K_{x}}=G^{S} G^{F_{x}}$.

Proof. (1) Notice that $K_{\chi} \subseteq K$ and $K \subseteq S$ by Proposition 1.2.

By Corollary 1.5, $G / \operatorname{Para}_{\chi}(G) \in K_{\chi}$. Therefore $G^{K_{\chi}} \leq \operatorname{Para}_{\chi}(G)$. Suppose there exists a $G$-chief factor $F=\operatorname{Para}_{\chi}(G) / N$ with $G^{K_{\chi}} \leq N$. By definition of $\operatorname{Para}_{\chi}(G)$, the chief factor $F$ is non- $\chi(G)$-complemented in $G$, and on the other hand, $F$ is $\chi(G)$-complemented in $G$ because $G^{K_{\chi}} \leq N$. Such $F$ cannot exist and $\operatorname{Para}_{\chi}(G)=G^{K_{x}}$.

(2) Let $G$ be a group such that $\mathrm{Q}(G) \subseteq \Re_{\chi}$. If $H / K$ is a $\chi$-Frattini chief factor of $G$ then $H \leq M$ for all $M \in \chi(G)$ such that $K \leq M$. That is to say that $H / K \leq \Phi_{\chi}(G / K)=1$, a contradiction. So $F_{\chi}=\left(G \in E: \mathrm{Q}(G) \subseteq \Re_{\chi}\right)$. The proof for $G^{F_{\chi}}=\operatorname{Pro}_{\chi}(G)$ is similar to (1).

(3) This is straightforward verification.

(4) The factorization is deduced directly from (3).

THEOREM 2.3. Let $\chi$ be a solid function satisfying the property that for each group $X$, if $Y$ is a normal subgroup of $X$ and $U \in \chi(X)$, and if $D$ is a normal subgroup of $Y$ such that $D(U \cap Y)$ is a maximal subgroup of $Y$, then $D(U \cap Y) \in \chi(Y)$. If every abelian chief factor of a group $G$ is $\chi$-complemented in $G$, then every abelian chief factor of a normal subgroup $N$ of $G$ is $\chi$-complemented in $N$. 


\section{A. BALLESTER-BOLINCHES, H. BECHTELL AND L. M. EZQUERRO}

Proof. Deny the statement and let $G$ be a group of minimal order such that every abelian chief factor of $G$ is $\chi$-complemented and there exists a normal subgroup $N$ of $G$ such that $N$ possesses a $\chi$-Frattini abelian chief factor.

Consider a $G$-chief series of $G$ through $N$ and refine the portion of this chief series under $N$ to obtain an $N$-chief series. Theorem A of [2] allows to say that there exists a $\chi$-Frattini abelian chief factor of $N$ in this series. Denote it by $T / L$. Assume that $T / L$ appears after refining the chief factor $H / K$ of $G$ under $N$. By the minimality of $G, K=1$ and $H$ is a minimal normal subgroup of $G$. Now $H$ is a direct product of minimal normal subgroups of $N$ (see Theorem A.4.13 of [6]). Again by Theorem A of [2], these minimal normal subgroups of $N$ in $H$ are $N$-isomorphic to the chief factors of $N$ under $H$ and then if one of them, $T / L$, is abelian, all of them are abelian. Hence $H$ is abelian. By Theorem A of [2], we can assume that $L=1$ and $T$ is a $\chi$-Frattini abelian minimal normal subgroup of $N$. Moreover $H=T \times D$ with $D$ a normal subgroup of $N$.

However there exists $M \in \chi(G)$ such that $G=M H$ and $M \cap H=1$. Since $H \leq N$, we have $N=(M \cap N) H=[(M \cap N) D] T$, and $(M \cap N) D$ is a maximal subgroup of $N$. Since $\chi$ is a solid function satisfying the property of the statement, we have $(M \cap N) D \in \chi(N)$. Then $T$ is $\chi$-complemented in $N$ leads to a contradiction.

Hence the statement is true and the theorem is proved.

CoROLLARY 2.4. If every abelian chief factor of a group $G$ is complemented in $G$ and $N$ is a normal subgroup of $G$, then every abelian $N$-chief factor is complemented in $N$.

In other words, the formations $\mathrm{F}$ and $\mathrm{K}$ are closed under taking normal subgroups.

Remarks. 1. It is rather easy to see that $\mathrm{K}$ is the class of all soluble $\mathrm{nC}$-groups, that is, the class of all groups $G$ such that every normal subgroup of $G$ is complemented in $G$. Christensen in [4] proved that $\mathrm{K}$ is a formation closed under taking normal subgroups. Hofmann in [10] extends this result to the class $\mathrm{E}$ of all $\mathrm{nC}$-groups. Independently, this result has been proved in [3].

2. With the above notation, we see that $E \cap S=F \cap S=K$. This validates the longstanding conjecture that a non-trivial nC-group $G$ is solvable if and only if each $G$-chief factor is complemented by a maximal subgroup. Notice that $E \neq F$ : the group $G=\operatorname{Aut}(\operatorname{Alt}(6))$ is not a nC-group and $G \in F$.

3. Partially nonsaturated formations. One well-known feature of a saturated formation $\mathrm{X}$ is that in each group $G$, every chief factor of the form $G^{X} / N$ is supplemented. Totally nonsaturated formations are defined in [5] as the formations $\mathrm{X}$ such that, in each group $G$, every chief factor of the form $G^{X} / N$ is Frattini. On the other hand, given a solid function $\chi$ in each group $G$, we see that every chief factor of the form $G^{K_{x}} / N$ is non- $\chi$-complemented.

The aim of this section is to define new types of formations which are extensions of the totally nonsaturated ones. They are not totally nonsaturated in the general universe.

Definition. Let $\chi$ be a solid function. A formation $X$ is said to be $\chi$-partially nonsaturated, a $\chi$-pn-formation, if for any group $G$, the residual $G^{X}$ is a $\chi$-parafrattini normal subgroup of $G$.

The Max-partially nonsaturated formations will be called simply partially nonsaturated formations. 
If $\mathrm{H}$ is a class of groups, the class $\mathrm{E}_{\mathrm{K}(x)}(\mathrm{H})=(G$ : there exists a normal subgroup $N$ of $G$ such that $G / N \in H$ and every chief factor of $G$ below $N$ is complemented by some maximal subgroup in $\chi(G)$ ). Similar arguments to those used in [5] show that $\mathrm{E}_{\mathrm{K}(\chi)}$ is a closure operation. Note that to verify the idempotence property, condition (b) in the definition of solid function plays an important role.

Since $\chi(G)$ is a solid set of maximal subgroups, $\chi(G)$ satisfies the JH-property in the sense of the first Remark of the paper. So arguments similar to those used in [5] prove that if $\mathrm{X}$ is a formation, then $\mathrm{E}_{\mathrm{K}(\mathrm{x})}(\mathrm{X})$ is also a formation.

Lemma 3.1. Let $\mathrm{X}$ be a formation and $\chi$ a solid function. Consider a group $G$, a $\chi$-prefrattini subgroup $W$ of the group $G$, and $N=\left\langle G^{X} \cap W^{g}: g \in G\right\rangle$. Then $G^{\mathrm{E}_{\mathrm{K}(x)}(X)} \leq N \leq G^{X}$.

Proof. Let $H / K$ be a chief factor of $G$ such that $N \leq K<H \leq G^{X}$. Since $W \cap G^{X} \leq N$, then $W$ avoids $H / K$. Therefore $H / K$ is $\chi$-supplemented in $G$. Assume that $H / K$ is non- $\chi$ complemented in $G$. Then there exists a maximal subgroup $M$ of $\chi(G)$ such that $G=M H$ and $K<M \cap H$. repeat the arguments of Theorem 1.8 of Section 1 to reach a contradiction. Hence $H / K$ is $\chi$-complemented in $G$. So $G / N \in \mathrm{E}_{\mathrm{K}(\chi)}(X)$. Then $G^{\mathrm{E}_{\mathrm{K}(x)}(X)} \leq N$.

For a solid function $\chi$, denote by $\mathcal{P}_{2}(\chi)$ the class of primitive groups of type 2 whose socle is $\chi$-complemented. In particular, $\mathcal{P}_{2}(\operatorname{Max})=\mathcal{P}_{2}{ }^{\prime}$ is the class of primitive groups of type 2 whose socle is complemented by a maximal subgroup of $G$. This type of primitive group is known as a primitive group with small maximal subgroups. Denote by $\mathcal{M}_{\Phi}$ the class of all monolithic groups $G$ such that $\operatorname{Soc}(G) \leq \Phi(G)$.

THEOREM 3.2. Let $\mathrm{X}$ be a formation and $\chi$ a solid function. The following statements are pairwise equivalent:

(i) $\mathrm{X}$ is $\chi$-partially nonsaturated.

(ii) $X=\mathrm{E}_{\mathrm{K}(\mathrm{x})}(X)$.

(iii) For all groups $G$ we have $G^{X}=\left\langle G^{X} \cap W^{g}: g \in G\right\rangle$ for each $W \in \operatorname{Pref}(\chi(G), G$.

(iv) $b(X) \cap \mathcal{P}_{2}(\chi)=\emptyset$ and for all groups $G$ we have $Z\left(G^{X}\right) \leq \Phi_{\chi}(G)$.

(v) $b(X) \subseteq \mathcal{M}_{\Phi} \cup\left(\mathcal{P}_{2} \backslash \mathcal{P}_{2}(\chi)\right)$.

Proof. (i) $\Rightarrow$ (ii). If $G \in \mathrm{E}_{\mathrm{K}(x)}(\mathrm{X}) \backslash \mathrm{X}$, then $G^{X} \neq 1$. Therefore there exists a chief factor $G^{x} / K$ non- $\chi$-complemented in $G$ and this is a contradiction.

(ii) $\Rightarrow$ (iii). Since $G^{\mathrm{E}_{(x)}(\mathrm{X})}=G^{X}$, it is clear by the previous lemma that $G^{X}=\left\langle G^{X} \cap W^{g}: g \in G\right\rangle$ for each $W \in \operatorname{Pref}(\chi(G), G)$.

(iii) $\Rightarrow$ (i). Assume that $G^{X} / K$ is a chief factor of $G$ complemented by a maximal subgroup $M \in \chi$ and $M$ belongs to a system of maximal subgroups $\mathcal{S}$. Then $W=W(G, \chi(G), \mathcal{S})$ avoids $G^{X} / K$ and thus $W \cap G^{X} \leq K$. Hence $\left\langle G^{X} \cap W^{g}: g \in G\right\rangle \leq K$ is a contradiction.

(ii) $\Rightarrow$ (iv). Use induction on $|G|$. If $Z\left(G^{X}\right)=1$, there is nothing to prove. Assume that $Z\left(G^{X}\right) \neq 1$ and consider minimal normal subgroup $N$ of $G$ contained in $Z\left(G^{X}\right)$. If $N$ is complemented in $G$ by a maximal subgroup $M \in \chi(G)$, then $G^{X}=\left(M \cap G^{X}\right) \times N$ and $M \cap G^{X}$ is a normal subgroup of $G$. Therefore $G^{X} /\left(M \cap G^{X}\right)$ is a chief factor of $G$ complemented by $M$ which implies $G /\left(M \cap G^{X}\right) \in \mathrm{E}_{\mathrm{K}(x)}(\mathrm{X})=\mathrm{X}$ and this is a contradiction. Hence $N \leq \Phi_{\chi}(G)$. Now consider $G / N$. We have $Z\left(G^{X}\right) / N \leq Z\left(G^{X} / N\right)=Z\left((G / N)^{X}\right)$ $\leq \Phi_{\chi}(G / N)=\Phi_{\chi}(G) / N$. Then $Z\left(G^{X}\right) \leq \Phi_{\chi}(G)$. 


\section{A. BALLESTER-BOLINCHES, H. BECHTELL AND L. M. EZQUERRO}

Furthermore if $X=\mathrm{E}_{\mathrm{K}(\chi)}(\mathrm{X})$ it is clear that $b(X) \cap \mathcal{P}_{2}(\chi)=\emptyset$.

(iv) $\Rightarrow$ (ii). Let $G \in \mathrm{E}_{\mathrm{K}(x)}(\mathrm{X}) \backslash \mathrm{X}$ of minimal order. Then $G \in b(X), G^{X}$ is the minimal normal subgroup of $G$, and $G^{X}$ is $\chi$-complemented in $G$. Since $G \notin \mathcal{P}_{2}(\chi), G^{X}$ is abelian. Hence $G^{X}=Z\left(G^{X}\right) \leq \Phi_{\chi}(G)$ is a contradiction.

(v) $\Rightarrow$ (i). Let $G$ be a group of minimal order such that $G^{X}$ is not $\chi$-parafrattini. There exists a normal subgroup $K$ of $G$ such that $G^{X} / K$ is a chief factor of $G$ complemented by a maximal subgroup $H \in \chi(G)$. By minimality, $K=1$ and $G^{X}$ is a minimal normal subgroup in $G$. If $N$ is a minimal normal subgroup of $G$ in core $G H$, we can find in $G / N$ the chief factor $(G / N)^{X}=G^{X} N / N$ complemented by $H / N$, a contradiction. Therefore $G$ is a primitive group in $b(X)$ whose socle is complemented by a maximal subgroup in $\chi(G)$, another contradiction. Hence $X$ is $\chi$-partially nonsaturated.

(i) $\Rightarrow$ (v). If $G \in b(X)$, then $G$ is a monolithic group whose minimal normal subgroup is $G^{X}$ and it is non- $\chi$-complemented in $G$. If $G^{X}$ is abelian, then $G \in \mathcal{M}_{\Phi}$. If $G^{X}$ is non-abelian, then $G \notin \mathcal{P}_{2}(\chi)$.

The case $\chi=$ Max is particularly interesting. We obtain a description of the partially nonsaturated formations.

THEOREM 3.3. Let $\mathrm{X}$ be a formation. The following statements are pariwise equivalent.

(i) $\mathrm{X}$ is partially nonsaturated.

(ii) $X=\mathrm{E}_{\mathrm{K}}(X)$.

(iii) For each group $G$ we have $G^{X}=\left\langle G^{X} \cap W^{g}: g \in G\right\rangle$ for each $W \in \operatorname{Pref}(G)$.

(iv) $b(X) \cap \mathcal{P}_{2}^{\prime}=\emptyset$ and, for all groups $G$ we have $Z\left(G^{X}\right) \leq \Phi(G)$.

(v) $b(X) \subseteq \mathcal{M}_{\Phi} \cup\left(\mathcal{P}_{2} \backslash \mathcal{P}_{2}^{\prime}\right)$.

This result is an extension of Theorem 4.2 in [9].

REMARK. We can also define the $\chi$-totally nonsaturated formations as the formations $X$ such that for any group $G$ every chief factor of the form $G^{X} / K$ is $\chi$-Frattini in $G$, or in other words, $G^{X}$ is a $\chi$-profrattini normal subgroup of $G$. The formation $F_{\chi}$ is an example of a $\chi$-totally nonsaturated formation. A description of this type of formations can be made just by the appropriate modifications of Theorem 5.3 of [1].

The group $G=\operatorname{Aut}(\operatorname{Alt}(6))$ is a primitive group of type 2 whose minimal normal subgroup is not complemented by any subgroup of $G$ : hence $G \in \mathcal{P}_{2} \backslash \mathcal{P}_{2}^{\prime}$. Furthermore $G / \operatorname{Soc}(G) \cong C_{2} \times C_{2}$. Consequently $G \in b(K)$. Nevertheless, the boundary of a totally nonsaturated formation does not contain primitive groups of type 2 . Therefore $\mathrm{K}$ is not a totally nonsaturated formation.

The group $G=\operatorname{Aut}(\operatorname{Alt}(6))$ is in the boundary of the formation $C$ of all $\mathrm{nC}$-groups. Hence, the class $\mathrm{C}$ is not a totally nonsaturated formation either.

ACKNOWLEDGEMENT. The authors thank the referee for his careful reading of the original paper and his suggestions that have improved this final version.

\section{REFERENCES}

1. A. Ballester-Bolinches and L. M. Ezquerro, On maximal subgroups of finite groups, Comm. Algebra. 19(8) (1991), 2373-2394. 
2. A. Ballester-Bolinches and L. M. Ezquerro, The Jordan-Hölder theorem and prefrattini subgroups of finite groups, Glasgow Math. J. 37 (1995), 265-277.

3. A. Ballester-Bolinches and M. D. Pérez-Ramos, Finite nC-groups. Preprint.

4. C. Christensen, Groups with complemented normal subgroups. J. London Math. Soc. 42 (1967), 208-216.

5. K. Doerk, Über Homomorphe und Formationen endlicher auflösbarer Gruppen (Habilitationschrift Mainz, 1971).

6. K. Doerk and T. O. Hawkes, Finite soluble groups De Gruyter Expositions in Mathematics, No. 4. (De Gruyter, 1992).

7. D. Gorenstein, Finite groups (Chelsea, New York, 1980).

8. U. C. Herzfeld, Frattiniclasses of formations of finite groups, Bol. Un. Mat. Ital. (7). 2-B (1988), 601-611. 199-207.

9. M. C. Hofmann, A residual generating prefrattini subgroup. Arch. Math. (Basel) 48 (1987), 5501 .

10. M. C. Hofmann, The normal complemented formation, Comm. Algebra. 23(14) (1995), 5499-

A. Ballester-Bolinches

Departament D'Algebra

Universitat De ValènCIA

C/Dr MOLINER 50

46100 BURJASSOT

VALÈNCIA

SPAIN

L. M. Ezquerro

Departamento de Matemática E Informática

Universidad Pública de Navarra

Campus de Arrosadia

31006 PAMPLONA

SPAIN
H. Bechtell

Department of Mathematics

Kingsbury Hall

UNIVERSITY OF NEW HAMPSHIRE

DURHAM

New Hampshire 03924

USA 